\title{
Modeling and Simulation of Primary Damage and Structure Evolution in Ceramics and Metals
}

\author{
Fei GAO \\ Dept. of Nucl. Eng. and Radiological Sciences, College of Engineering, University of Michigan (Ann Arbor, Michigan,USA)
}

Understanding the basics of ion-solid interaction and irradiation damage has led to significant developments in state-of-the-art atomic-level, kinetic Monte Carlo and meso-scale simulations, and these simulations have dramatically advanced the understanding of defect and defect processes in a number of materials, ranging from metals to semiconductors to insulators. Recent progress in multiscale simulations of fundamental ion-solid interactions, defect generation and defect evolution in ceramics and metals is reviewed, which will provide significant insights into the radiation resistance behavior of these materials.

Recently, large-scale ab initio MD simulation methods have been developed for the study of radiation damage in ceramics [1,2], and these methods are used to explore the effects of charge transfer and charge-density redistribution on the dynamics and charge-state of defect formation. We will focus on the applications of silicon carbide ( $\mathrm{SiC}$ ) because of its a number of understanding physical and chemical properties that lead to its potential applications to semiconductor devices, nuclear reactors for power generation and petrochemical industries, particularly under severe environments. The small cross section, low activation and good thermal conductivity of SiC under neutron irradiation lead to its potential use in structural components for fusion reactor or nuclear waste technology, and as cladding material for gas-cooled fission reactors. Fig. 1(a) shows a perfect structure of 3C-SiC, where large spheres represent $\mathrm{Si}$ atoms and small spheres indicate $\mathrm{C}$ atoms. Figs. 1 (b) and (c) show the charge density of the final configuration of a $C$ recoil (b) or a Si recoil (c) along the $\{111\}$ direction in the (110) plane. In Fig. 1(b), the most interesting observation is that some charge accumulates on the $\mathrm{C}$ vacancy, mainly transferred from the C PKA. The charge is mostly distributed in the region between the $\mathrm{C}$ vacancy and one of its neighboring $\mathrm{Si}$ atoms, suggesting a tendency for the $\mathrm{C}$ vacancy pairing with the $\mathrm{Si}$ atom. Obviously, this $\mathrm{C}$ vacancy is a positively-charged vacancy. For the Si recoil, the charge density shows that the Si tetrahedral interstitial forms new bonds with its nearest $\mathrm{C}$ atoms, as shown by the charge accumulation in the bonding regions (Fig. 1(c)). However, the $\mathrm{Si}_{\mathrm{TC}}$ prefers to form a new bond with the Si atom below (indicated by an arrow). A strong bond formed between the $\mathrm{Si}_{\mathrm{TC}}$ and its nearest $\mathrm{C}$ atom may facilitate the charge transfer between the two nearby $\mathrm{Si}$ atoms, leading to charge accumulation in the bonded regions and the formation of a new covalent bond. Fig. 1(c) also clearly shows that the Si vacancy does not pair with its neighboring atoms, and is in neutral state.
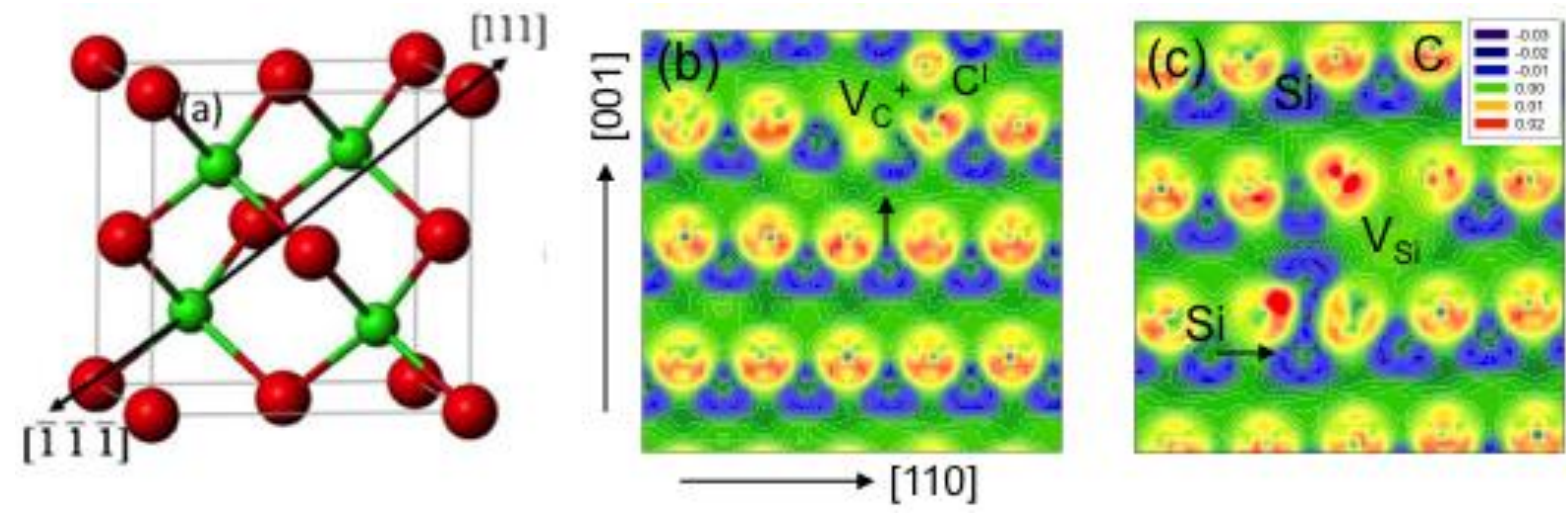

Fig. 1: Perfect crystal of (a) SiC (small spheres-C atoms and large spheres-Si atoms), chargedensity difference contour plot for a $C$ recoil (a) and a Si recoil (b) along the $<\overline{1} \overline{1}>$ direction in a $(1 \overline{1} 0)$ plane (in unit of $|e| / a_{b}{ }^{3}$, where $a b$ is Bohr radius). 
Next atomic-level study of radiation damage is molecular dynamics simulation that has been approved to be sufficiently powerful that many phenomena associated with radiation damage effects in metals and ceramics. Recent studies of several such phenomena in metals and SiC are reviewed here. In metals, at high enough recoil energy, they create cascades of atomic displacements that result in single and clustered self-interstitial and vacancy defects. These studies provide data on the number of defects produced and their distribution in clusters. I will focus on the formation of defect clusters and dislocation loops. Furthermore, molecular dynamics (MD) simulations to determine defect configurations, defect production, cascade-overlap effects, and defect migration energies and pathways will be reviewed in both bulk $\mathrm{SiC}$ and plycrystaslline $\mathrm{SiC}$. The role of interfacial processes in ceramic-ceramic composites will also be discussed. Factors that can improve the radiation tolerance of ceramics will be highlighted. Overlapping of $\mathrm{Si}$ cascades results in the interaction of defects and clusters that stimulates cluster growth and produces long-range structural disorder. Highresolution TEM images, disordering behavior, volume change and mechanical properties obtained experimentally and from molecular dynamics simulations are in good agreement, thus providing atomic-level interpretation of experimentally observed features [3].

In terms of polycrystalline $\mathrm{SiC}$, it is of interest to note that the mechanisms of defect generation in nanocrystalline (NC) $\mathrm{SiC}$ are found to be different from those observed in metals. However, for average grain diameters larger than $20 \mathrm{~nm}$, most defects are produced within nano grains, rather than inside the grain boundaries in NC SiC. The defect production within grains generally increases with increasing grain size, which is manifested in switching from grain boundary damage to grain damage. The most common defects created in NC SiC are antisite defects, following by vacancies and interstitials, in contrast to those produced in a single crystalline $\mathrm{SiC}$, where the dominate defects are Frenkel pairs. These simulations provide important insights into how nanostructures influence the radiation response or tolerance of ceramics.

A kinetic lattice Monte Carlo (KLMC) model has been developed to investigate the long-time scale simulations associated the recovery and clustering of defects during annealing of a single $10 \mathrm{keV}$ cascade in cubic silicon carbide [4]. The $10 \mathrm{keV} \mathrm{Si} \mathrm{cascade} \mathrm{is} \mathrm{produced} \mathrm{by} \mathrm{MD,} \mathrm{and} \mathrm{a} \mathrm{method} \mathrm{of}$ transferring the defects created by MD simulations to the KLMC model is developed. The KLMC model parameters are obtained from MD simulations and ab initio calculations of defect migration, recombination, and annihilation. The defects are annealed isothermally from $100 \mathrm{~K}$ to $1000 \mathrm{~K}$ in the KLMC model. Two distinct recovery stages for close Frenkel pairs are observed at about 200 and $550 \mathrm{~K}$, and the growth of complex clusters is observed above $400 \mathrm{~K}$. These simulation results are in good agreement with available experimental results.

\section{References}

[1] F. Gao, H. Y. Xiao, X. T. Zu, M. Posselt, W. J. Weber, "Defect-Enhanced Charge Transfer by Ion-Solid Interactions in SiC using Large-Scale Ab Initio Molecular Dynamics Simulations", PRL 103 (2009) 027405.

[2] F. Gao, H. Y. Xiao, W. J. Weber, "Ab Initio Molecular Dynamics Simulations of Low Energy Recoil Events in Ceramics", Nuclear Instruments and Methods in Physics Research. Section B, Beam Interactions with Materials and Atoms 269 (2011) 1693.

[3] F. Gao, WJ Weber, "Mechanical Properties and Elastic Constants due to Damage Accumulation and Amorphization in SiC" Phys. Rev. B 69 (2004) 224108.

[4] Z. Rong, F. Gao, and W. J. Weber, "Monte Carlo Simulations of Defect Recovery within a $10 \mathrm{keV}$ Collision Cascade in 3C-SiC", Journal of Applied Physics 102 (2007) 103508. 


\section{Modeling and Simulation of Primary Damage and Structure Evolution in Ceramics and Metals}

\section{Fei Gao}

Department of Nuclear Engineering and Radiological Sciences, University of Michigan, Ann Arbor, MI 48109 USA 


\section{Outline}

$>$ Introduction - radiation damage, defects and microstructure evolution

$>$ Metals

- Defect production - different structures (bcc, fcc, hcp), temperature.

- Defects, defect clustering and effects of interatomic potentials

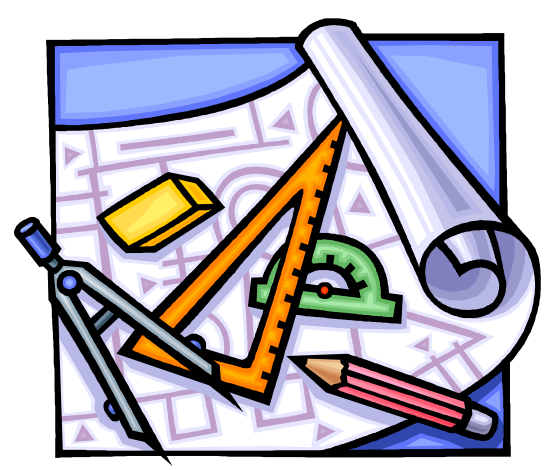

- Defect production under different conditions - pre-strained crystal, grain boundaries and dislocations

$>$ Ceramics

- $\quad$ Ab initio MD simulations of defect generation - threshold energy, charge defects and charge-enhanced migration of defects

- Defect generation and clustering, defect accumulation and mechanical properties

$>$ Further consideration

- Kinetic Monte Carlo, phase field modeling and cluster dynamics 


\section{Damage Evolution}

- damage accumulation (swelling, hardening, creep, ...) requires longer times, investigate with other methods

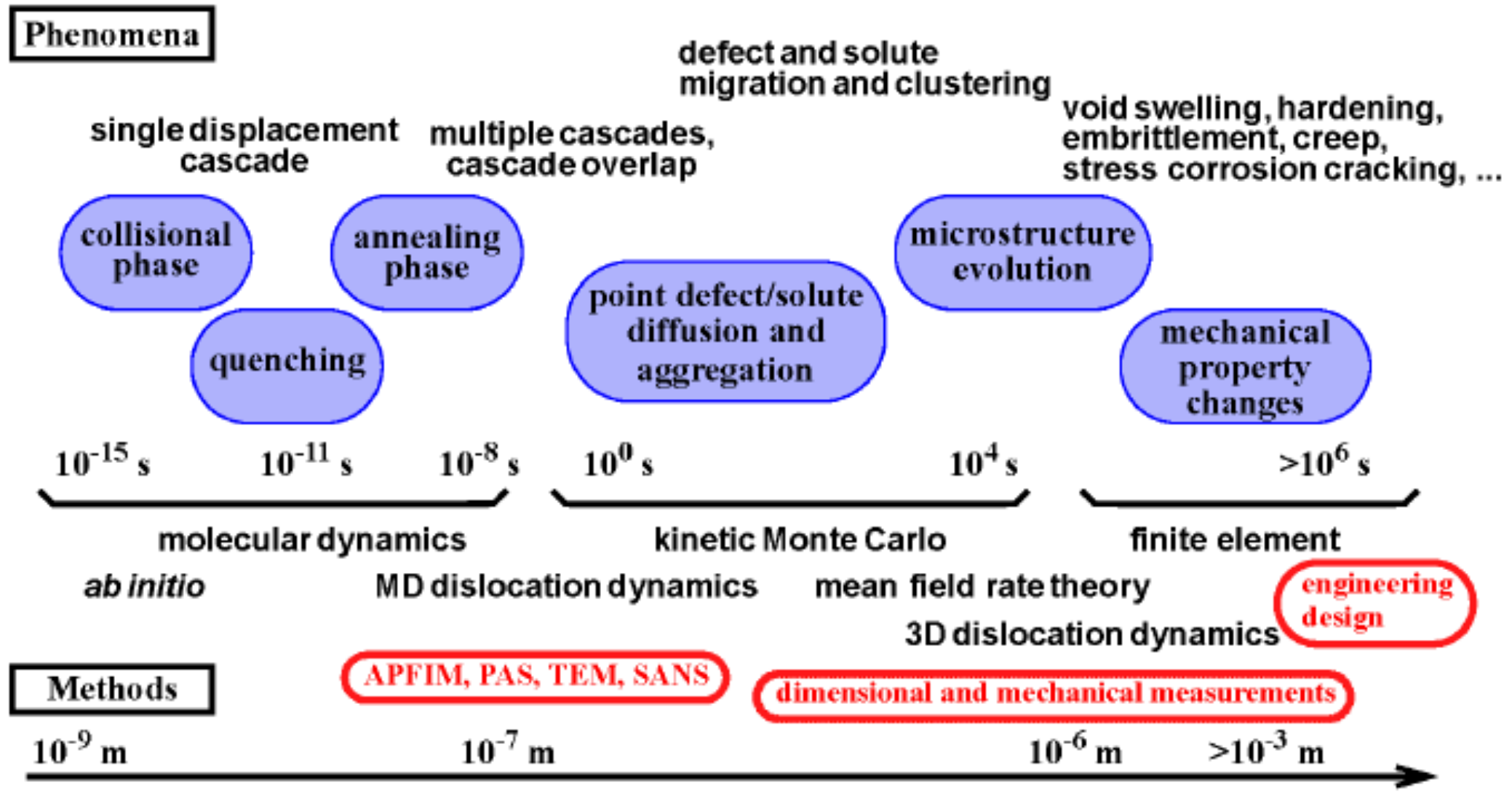

- need to maintain fidelity to physics in spite of approximations necessary for longer time/length scale methods 


\section{Defect Production in Different Structures}

$>\quad b c c-10 \mathrm{keV}, 100 \mathrm{~K}$ in $\mathrm{Fe}$

Www.liv.ac.uk/ afcalder

The University of Liverpool

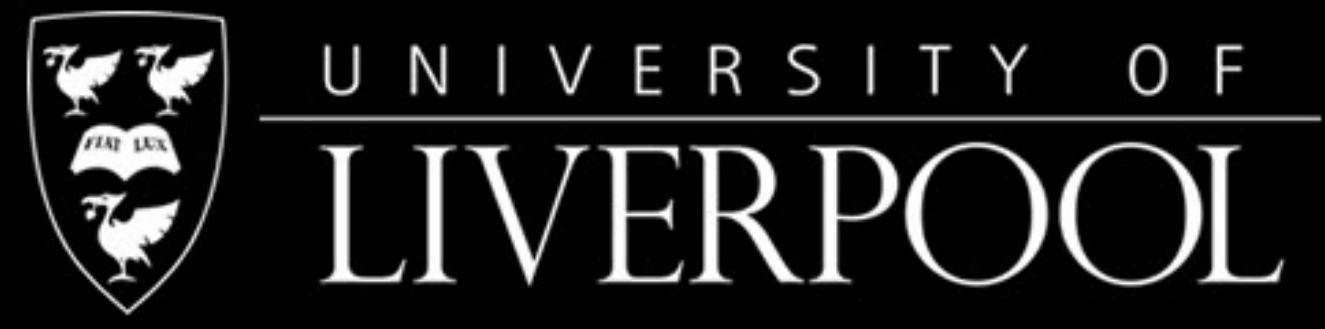

$>$ Most of the defects are single defects.

Acknowledgement: The Materials Modelling Group and The University of Liverpool UK 


\section{Defect Production in Different Structures}

$>\mathrm{fcc}-25 \mathrm{keV}, 100 \mathrm{~K}$ in $\mathrm{Cu}$

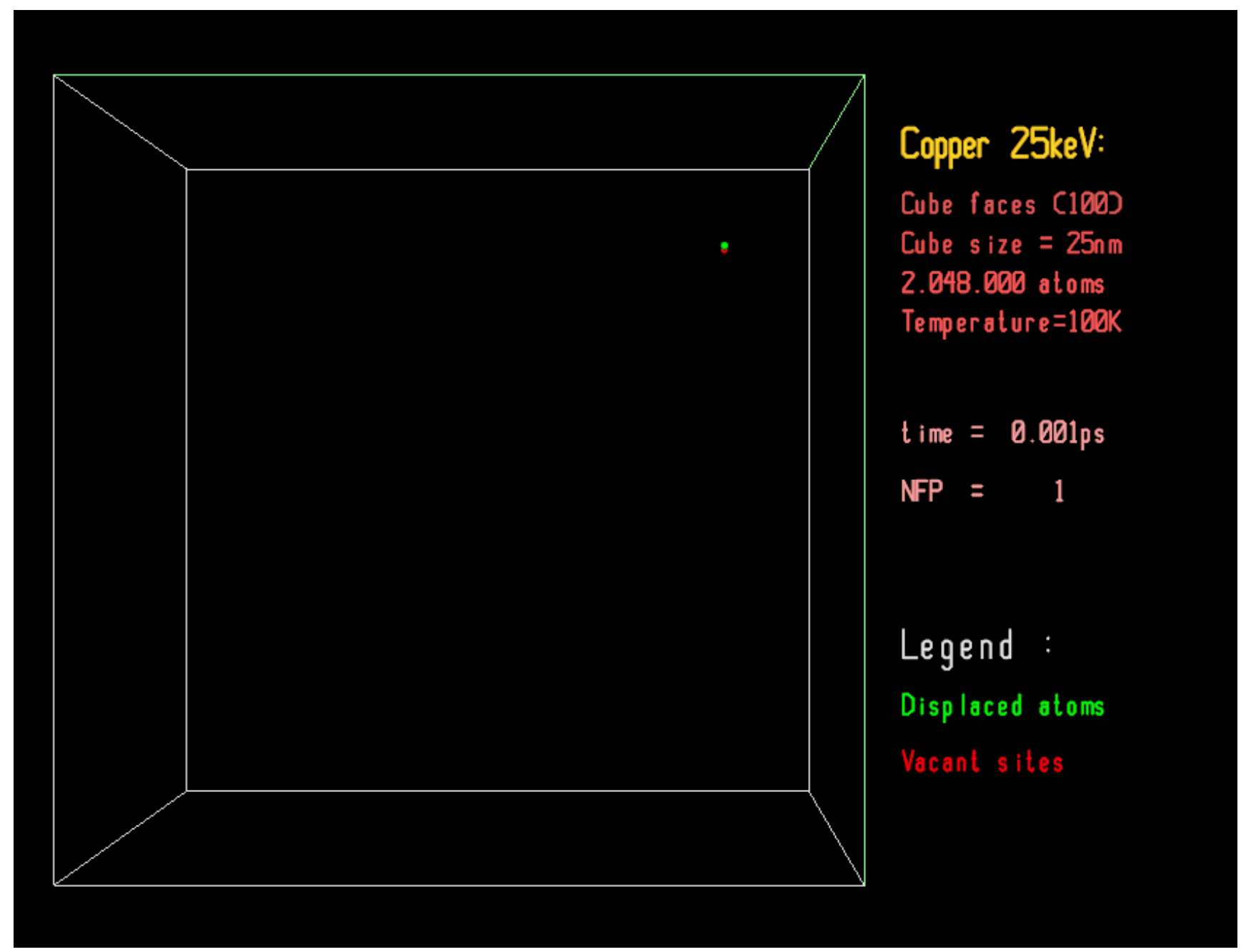

$>$ Different sizes and types of clusters are created.

$>$ A large vacancy cluster is created (tetrahedral stacking fault )

Acknowledgement: The Materials Modelling Group and The University of Liverpool UK 


\section{Defects and Defect Clustering in bcc, fce and hep}

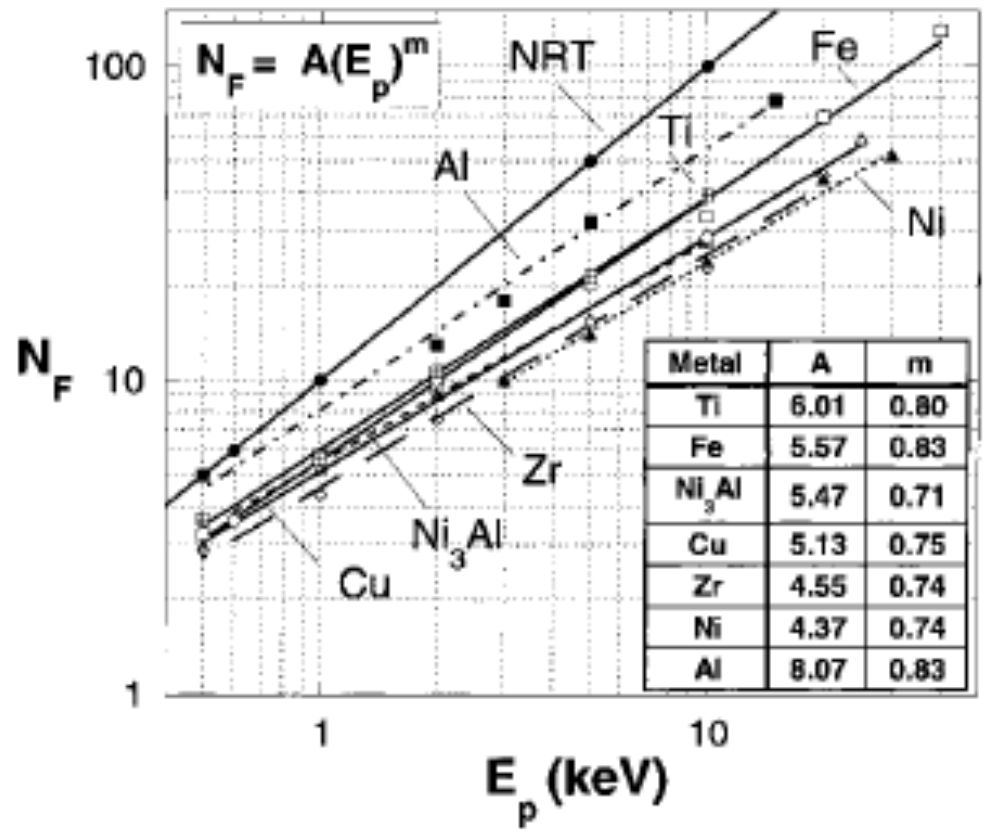

Fig. I, Log-log plots of $N_{\mathrm{F}}$ vs $\mathrm{E}_{\mathrm{p}}$ for $\mathrm{Ni}_{3} \mathrm{Al}$ and the pure metals $\mathrm{Cu}, \mathrm{Fe}, \mathrm{Ti}$ and $\mathrm{Zr}$ at $100 \mathrm{~K}$, and $\mathrm{Al}$ and $\mathrm{Ni}$ at $10 \mathrm{~K}$, demonstrating the power-law dependence of Eq. (2) in the text. The inset table shows the values of $m$ and $A$ (with $E_{p}$ in $\mathrm{keV}$ ) obtained with the best-fit lines shown in the figure. The data for $\mathrm{Al}$ and $\mathrm{Ni}$ were kindly supplied by Almazouzi et al. [20].

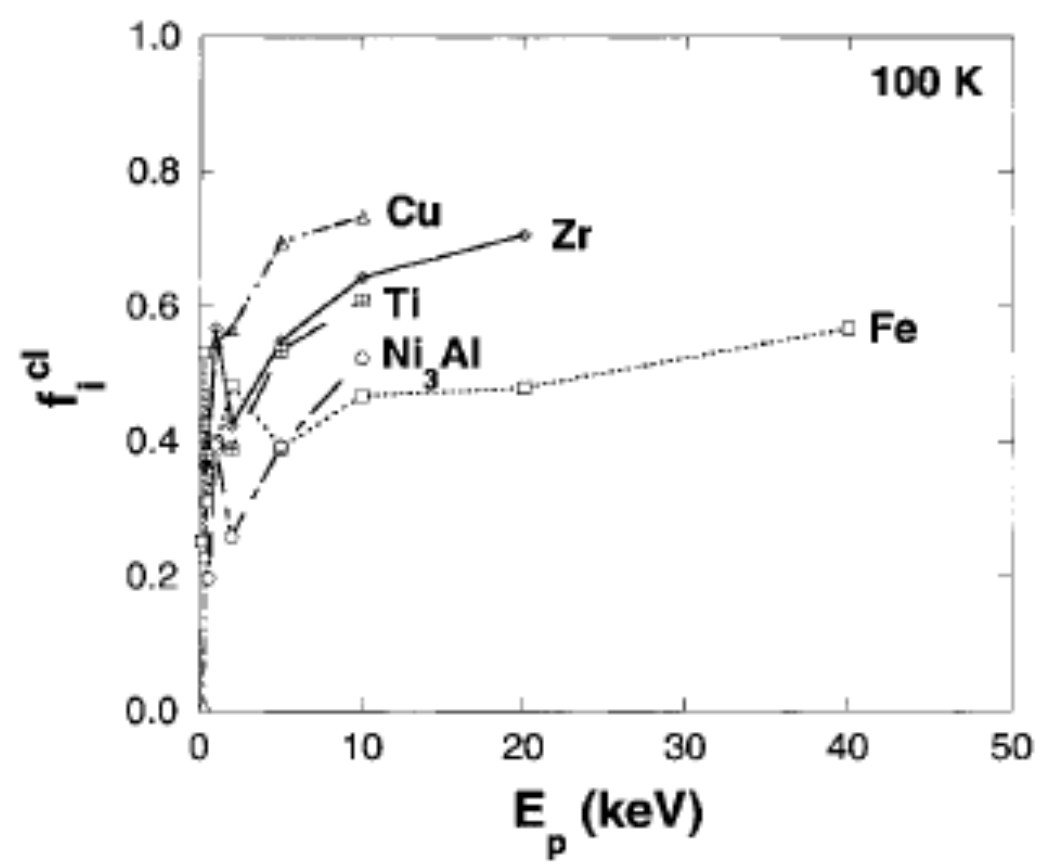

Fig. 3. The fraction, $f_{i}^{\text {ed }}$, of SIAs that survive in clusters of two or more in $\mathrm{Cu}, \alpha-\mathrm{Fe}, \alpha-\mathrm{Ti}, \alpha-\mathrm{Zr}$ and $\mathrm{Ni}_{3} \mathrm{Al}$ at $100 \mathrm{~K}$.

Bacon, et al, JNM 276, 2000

- different values, but similar power-law depending in defect survival - energy dependence of SIA clustering similar, varies by a factor $<2$ 


\section{Defects and Defect Clustering in bec, fec and hep}

$>$ Different $\mathrm{Fe}$ interatomic potentials
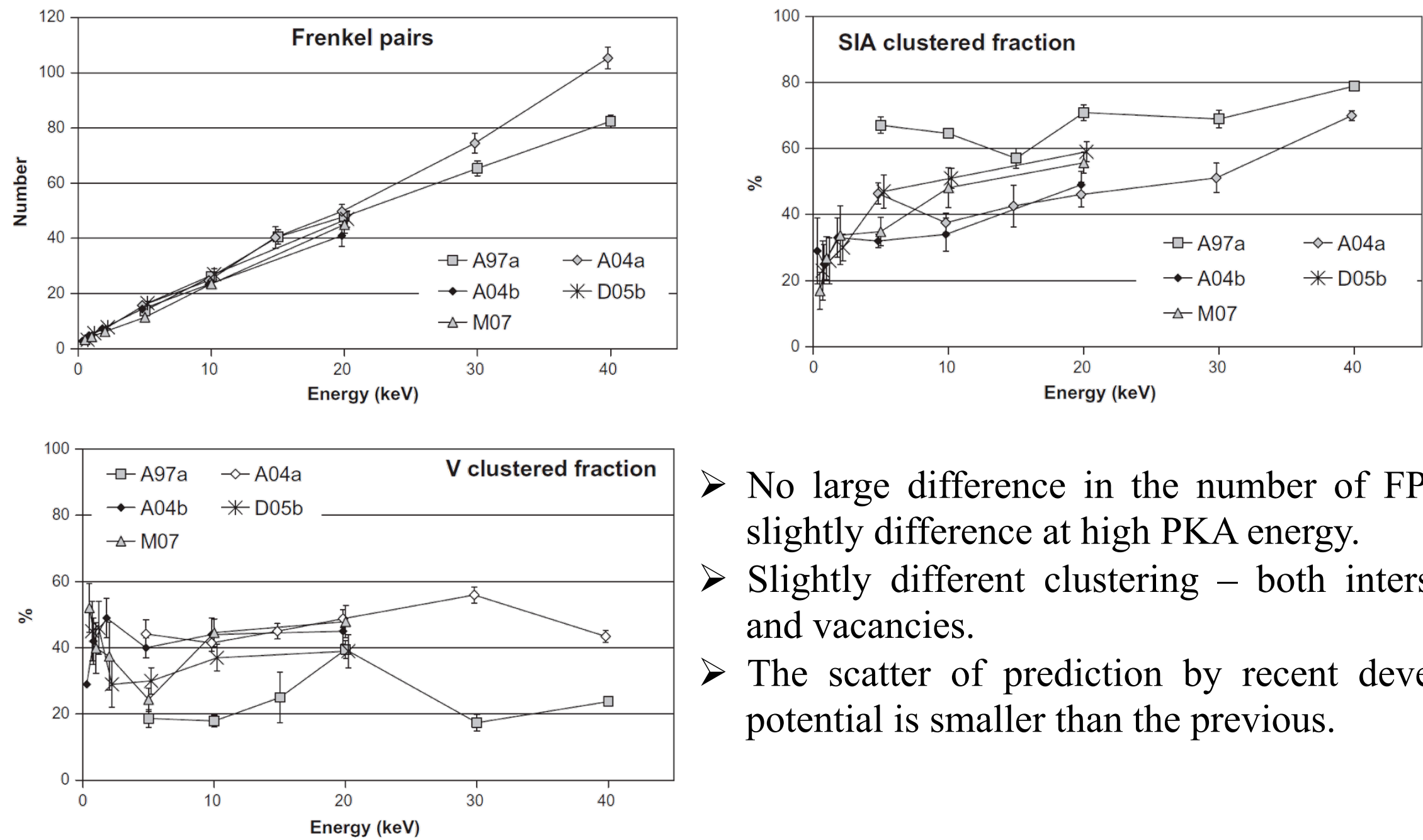

No large difference in the number of FPs, but slightly difference at high PKA energy.

$>$ Slightly different clustering - both interstitials and vacancies.

The scatter of prediction by recent developed potential is smaller than the previous.

L. Malerba et al., J. Nucl. Mater. 406 (2010) 19. 


\section{Displacement Cascades near GB in $\mathrm{Cu}$}

$\square$ Recent work on Cu (X. M. Bai et al. - Science 327 (2910) 1631)
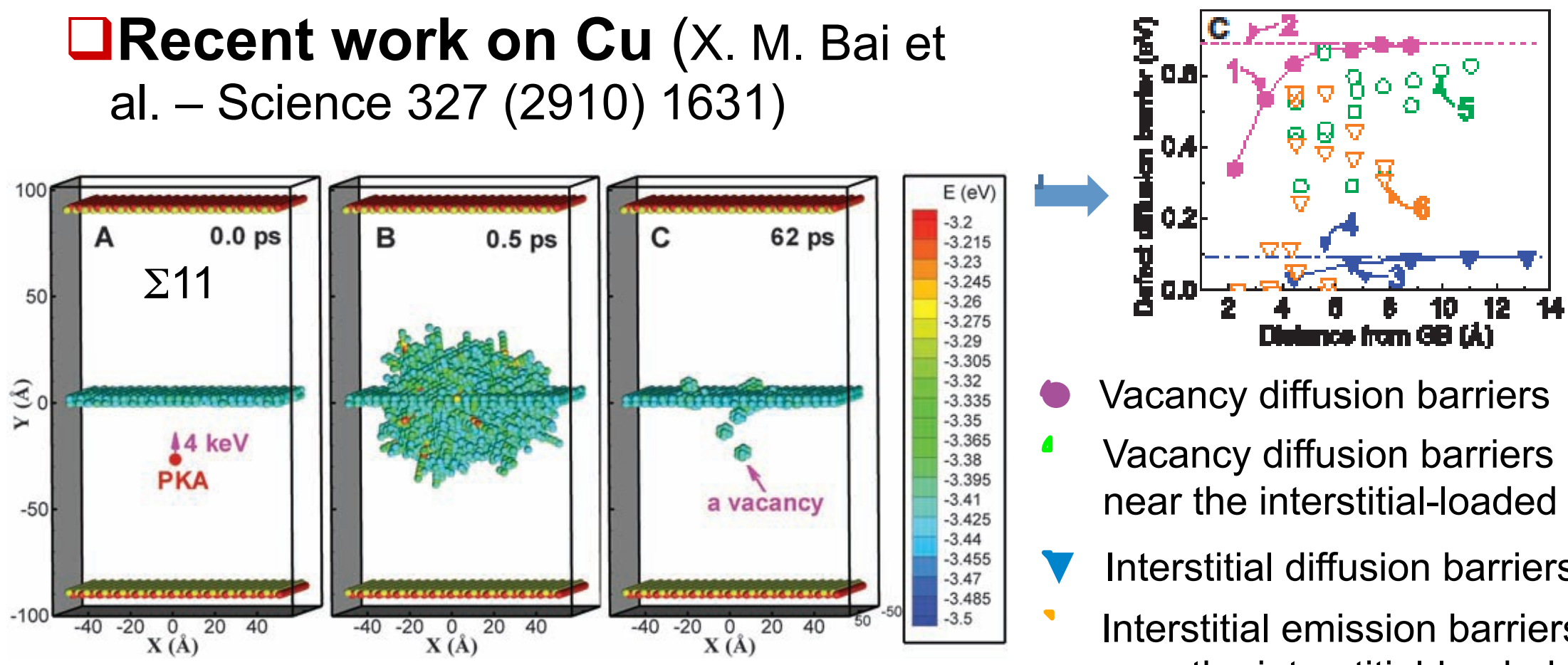

- Vacancy diffusion barriers near GB

- Vacancy diffusion barriers near the interstitial-loaded GB

$\nabla$ Interstitial diffusion barriers near GB

- Interstitial emission barriers near the interstitial-loaded GB

$>\mathrm{GBs}$ - a surprising "loading-unloading effect.

$>$ Interstitial can load into the GB, which acts as a source, emitting interstitials to annihilate vacancies in the bulk.

$>$ This is efficient for annihilating immobile vacancies in the nearby bulk. 


\section{Pre-strain Effect on Cascades in Zr}

\section{$>$ Zirconium Strain Effects}

Local strain in zirconium near hydride could be very high (up to $6 \%$ )
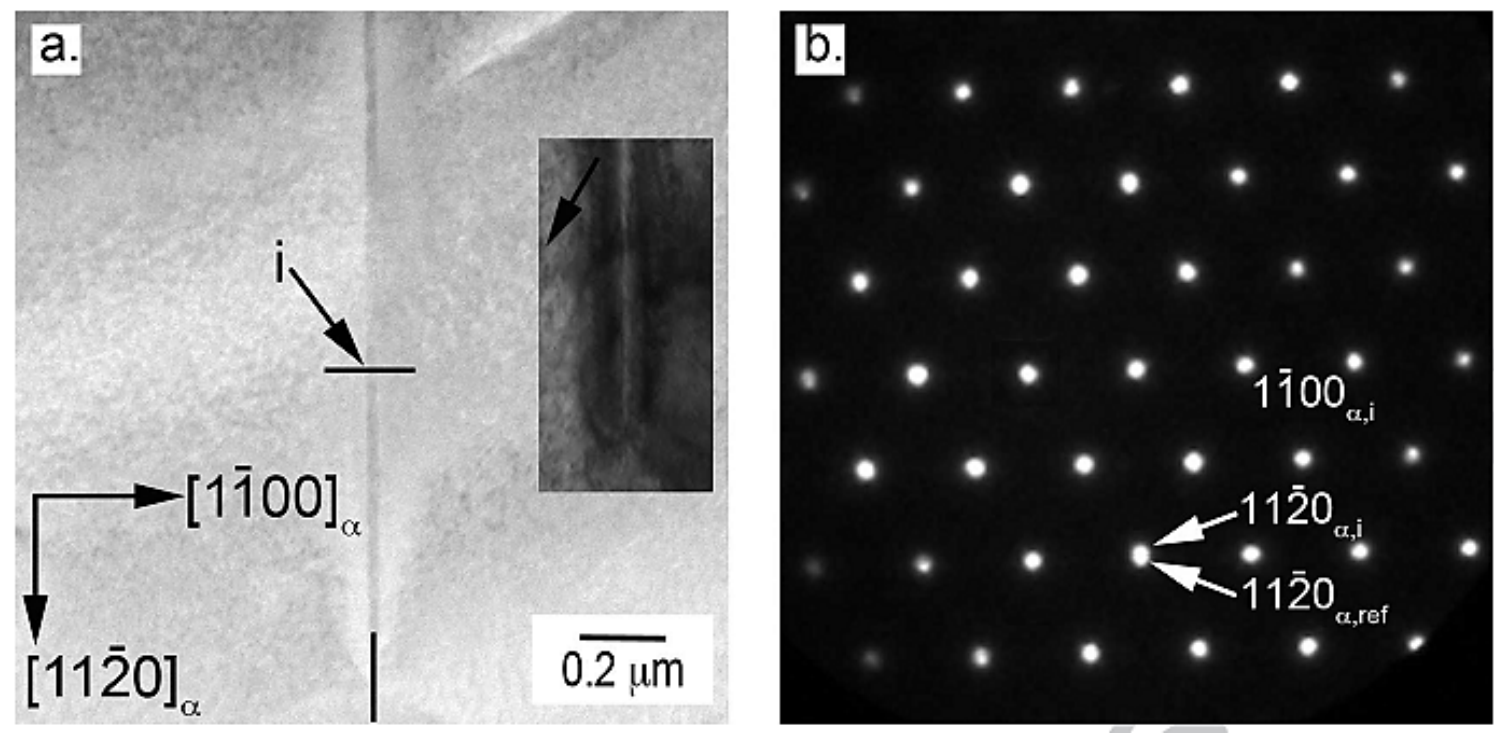

A.T.W. Barrow, A. Korinek, M.R. Daymond, J. Nucl. Mater., 432, 366 (2013).

$>$ A large number of voids are formed at the interface under irradiation a.
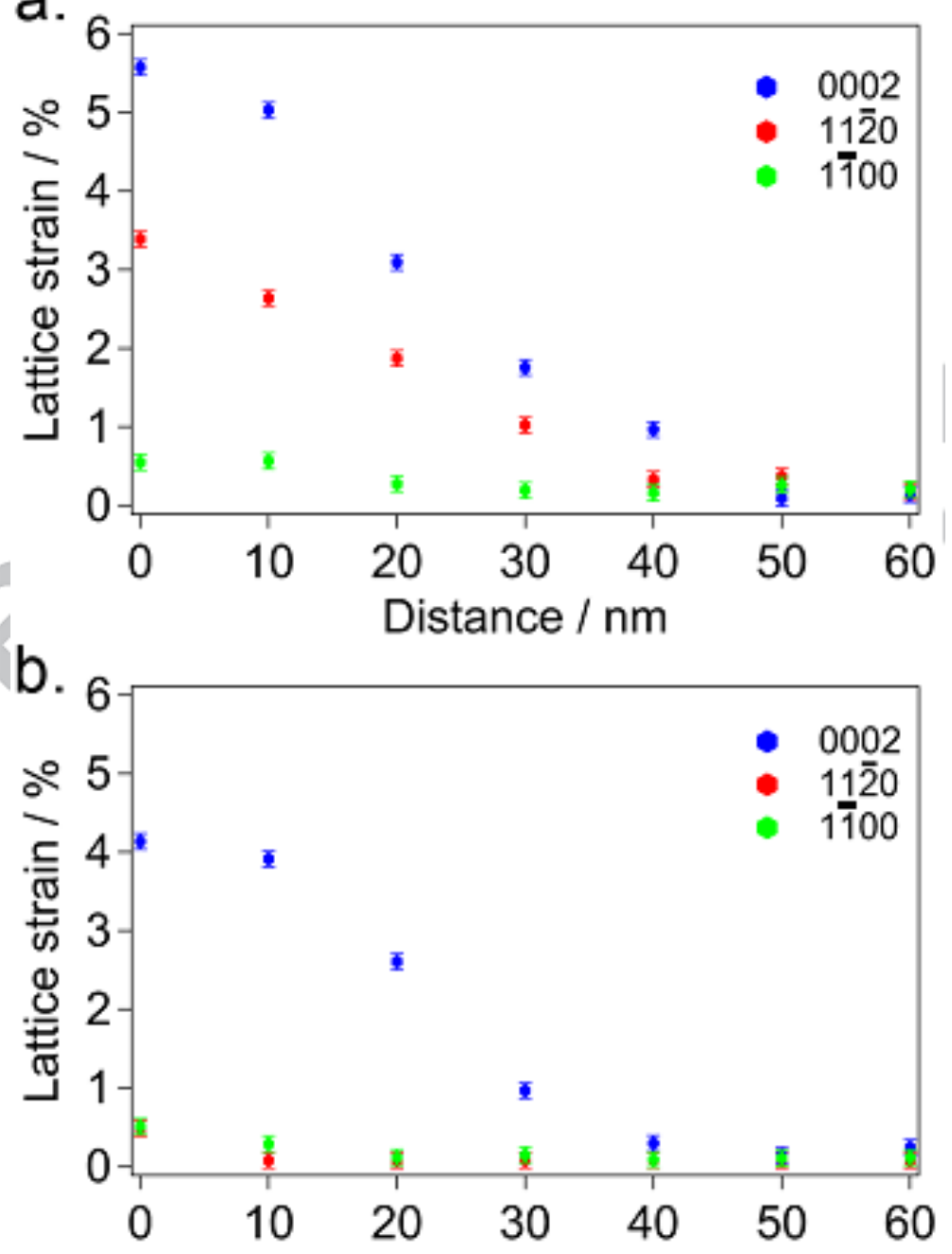


\section{Pre-strain Effect on Cascades in Zr}

\section{$\square$ Strain Effects (2-D video)}

$5 \%$ uni-axial strain along c-axial, $10 \mathrm{keV}$

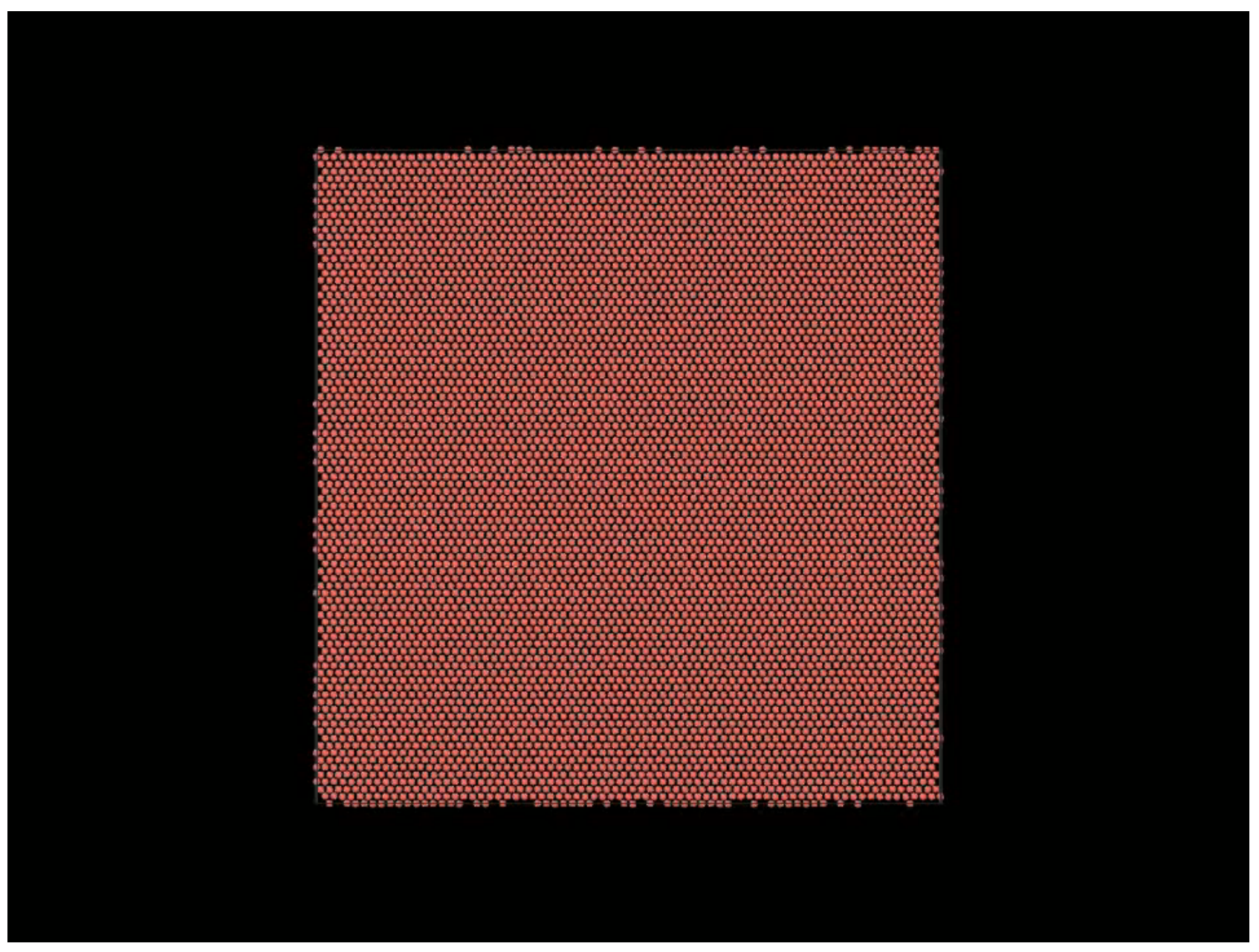

$>$ Void formation at the core of damage region, along with the emission of dislocations

$>$ Dislocations transport atoms from damage region

$>$ Dislocation accelerate void formation 


\section{Cascade Interaction with Dislocation}

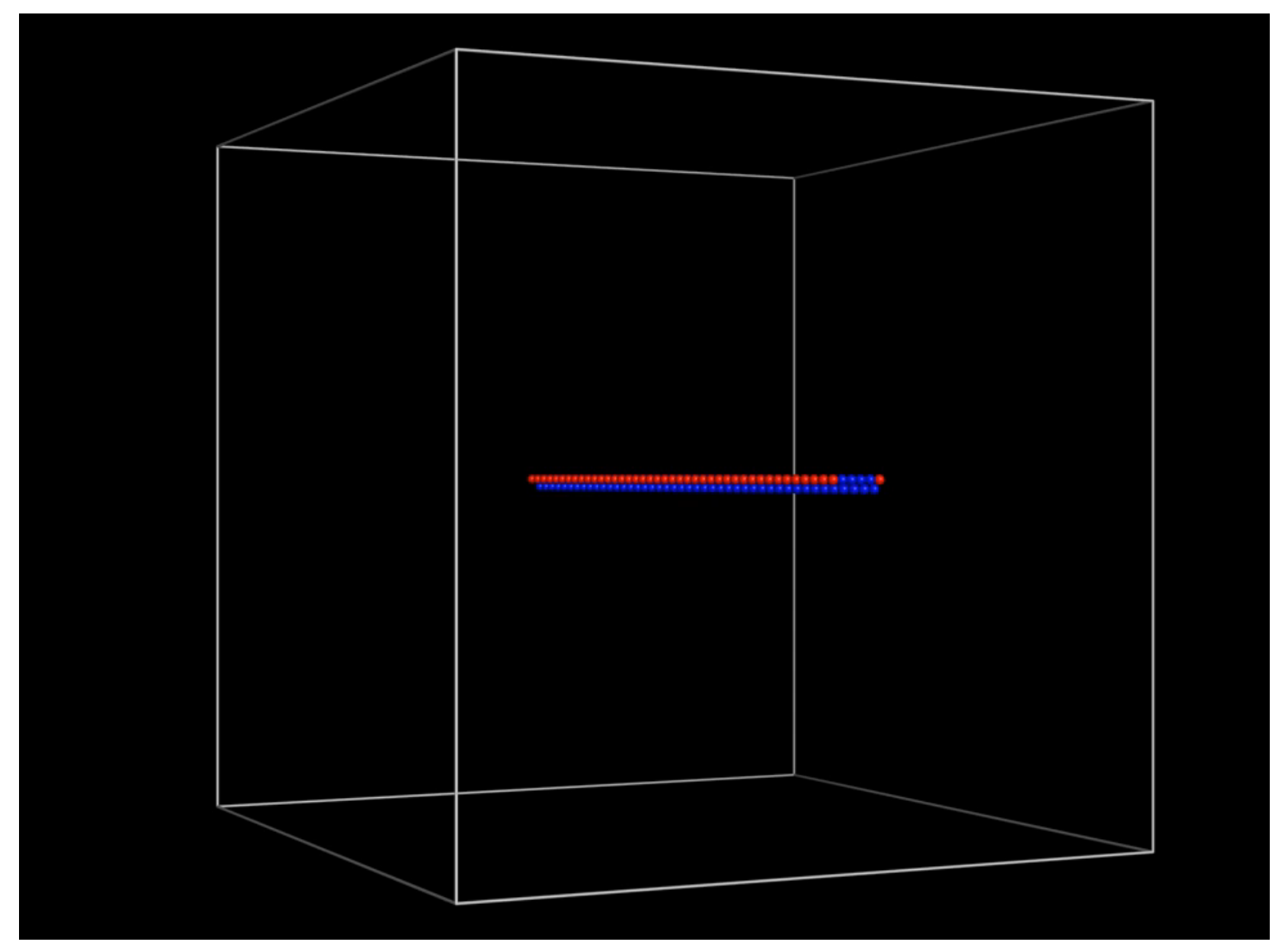

Formation of stacking fault tetrahedron near the dislocation line.

R.E. Voskoboinikov, Nucl. Instr. Meth. Phys. Res. B 303 (2013) 125-128. 


\section{Primary Damage in Ceramics}

$\square$ Large-scale ab initio MD simulation of defect creation $\sim \mathrm{E}_{\mathrm{d}}$

$>$ SIESTA and NWCHEM codes

> SIESTA - basis set: linear combination of numerical atomic orbitals (LCAO)

$>$ NWCHEM - basis set: plane wave

> Ion-electron interaction: normconserving Troullier-Martins pseudopotential

> Number of atoms -128 or 1000 atoms.

。

$>$ We have further developed the SIESTA code to include:

Initiate PKA at a given energy and a recoil direction

Conservation of energy and momentum

Variable time steps ( $1.0 \mathrm{fs} \sim 0.001 \mathrm{fs}$ ), which is based on energy and displacement criteria

$>\mathrm{SiC}, \mathrm{Y}_{2} \mathrm{Ti}_{2} \mathrm{O}_{7}, \mathrm{GaN}$, zirconate pyrochlores $\left(\mathrm{Nd}_{2} \mathrm{Zr}_{2} \mathrm{O}_{7}, \mathrm{Sm}_{2} \mathrm{Zr}_{2} \mathrm{O}_{7}, \mathrm{La}_{2} \mathrm{Zr}_{2} \mathrm{O}_{7}\right)$, $\mathrm{Gd}_{2} \mathrm{Zr}_{2} \mathrm{O}_{7}, \mathrm{Gd}_{2} \mathrm{Ti}_{2} \mathrm{O}_{7}, \mathrm{ThO}_{2}, \mathrm{CeO}_{2}, \mathrm{ZrO}_{2}$ and $\mathrm{SrTiO}_{3}$ 


\section{Primary Damage in Ceramics}

\section{$\square$ C [-1-1-1] Recoil}

$45 \mathrm{eV}$

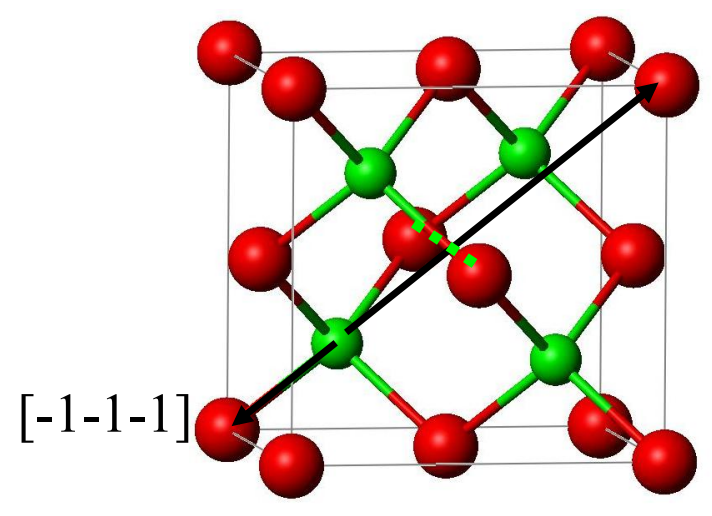

[111] PRL 103 (2009) 027405


$>$ Strong back recoil - leading to the formation of a C-C split interstitial 


\section{Primary Damage in Ceramics}

$\square$ C [-1-1-1] Recoil

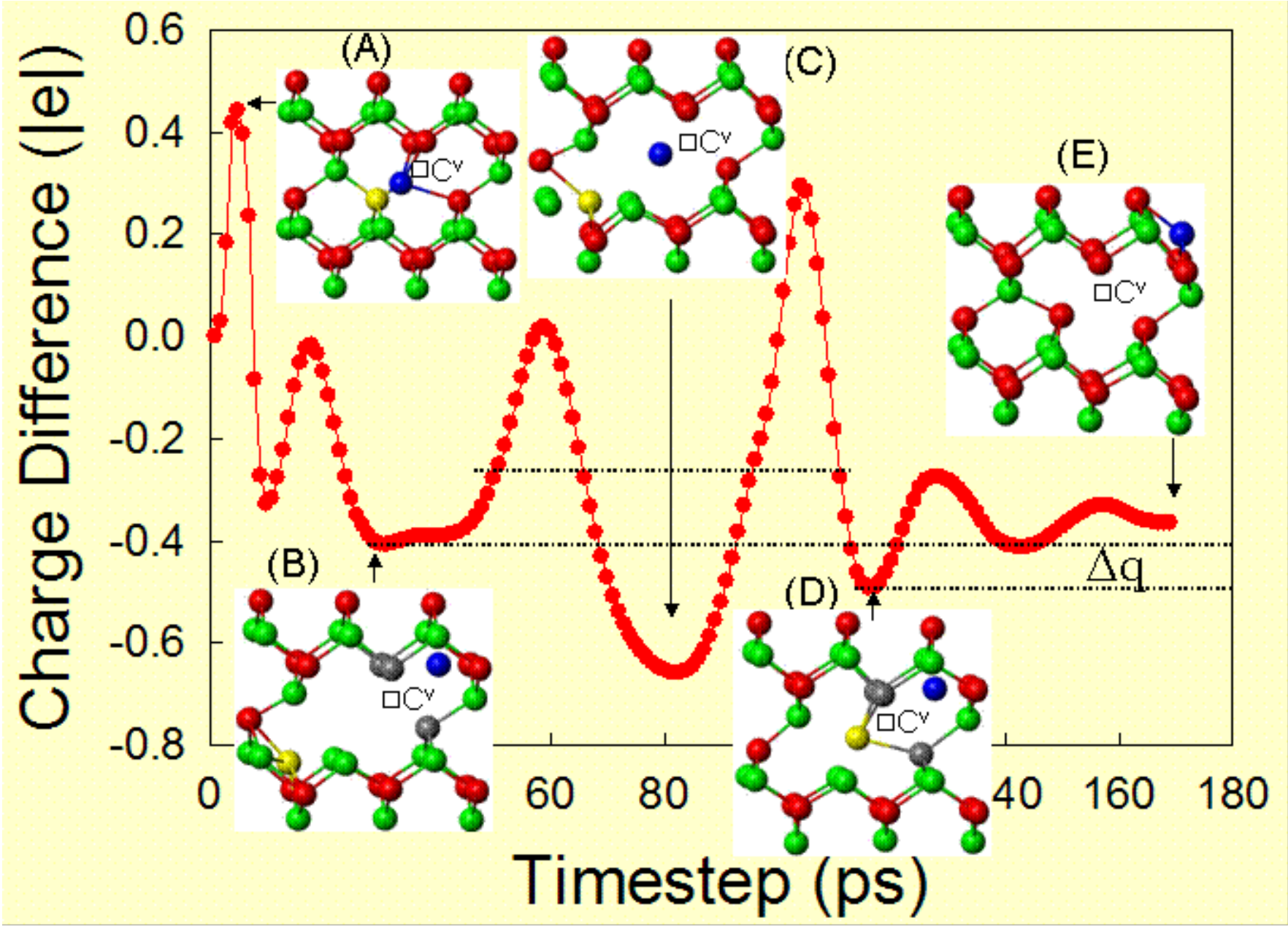

$>$ Note that the charge state in (B) is different from that in (D) $-\Delta \mathrm{q}$ 


\section{Primary Damage in Ceramics}

$\square \quad$ a C recoil $-47.5 \mathrm{eV}$
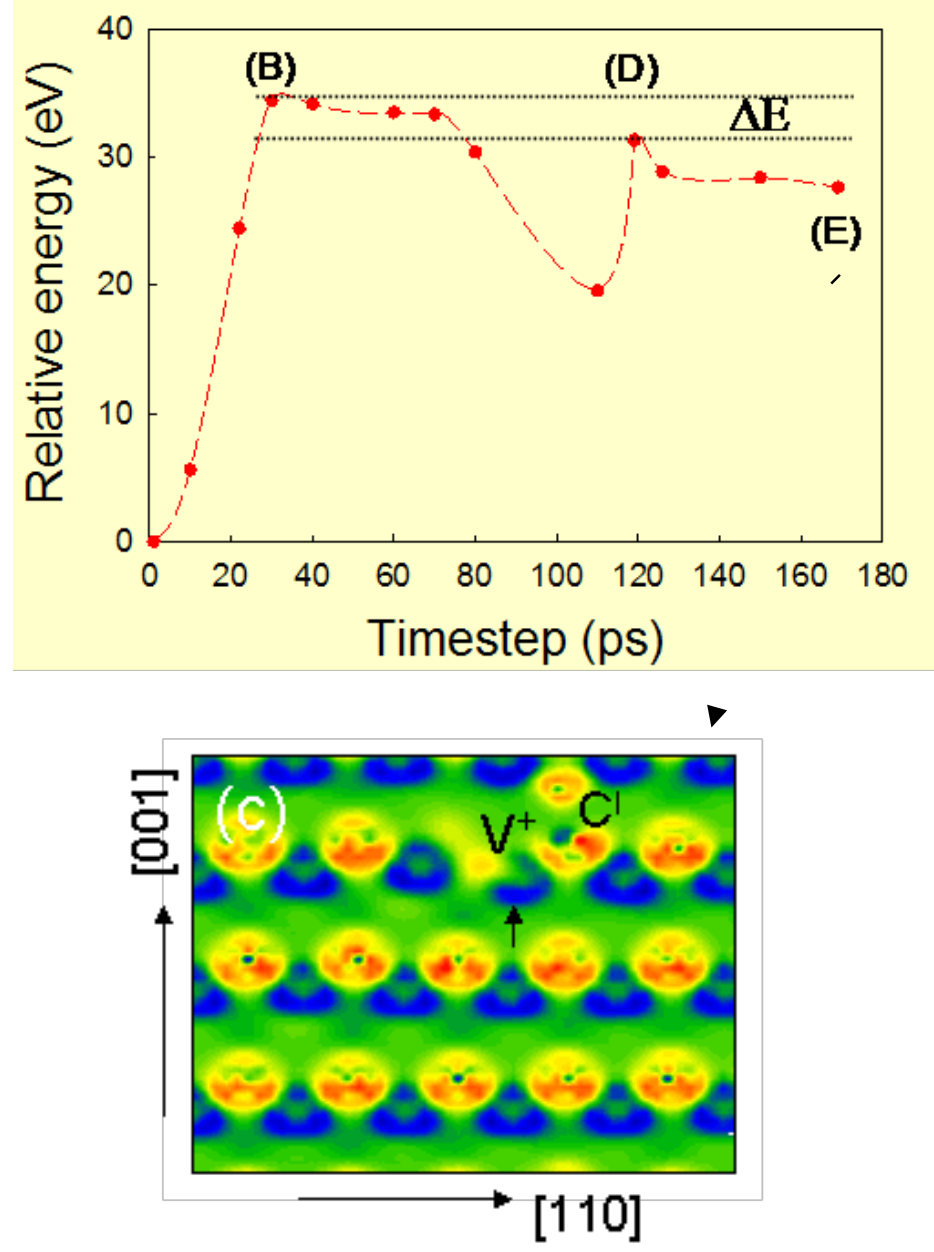

Charge-density contour plot for the final atomic configuration $(E)$ in a (1-10) plane
$>$ A charge-driven formation of defects important for covalent and ionic materials

- Some charges accumulate on the $\mathrm{C}$ vacancy

- Accumulated charge is not in the center of the vacancy, and the strong interaction of the charged vacancy with neighbors leads to a significant Jahn-Teller distortion

- C vacancy is in a positively charged state (fractional charges)

- All the results are consistent with previous experimental observations

- Present simulations show a dynamic process of how a charged defect is formed 


\section{Primary Damage in Ceramics}

$\square$ MD simulation of defect accumulation in $\mathrm{SiC}$

- The cascade overlap is simulated in a block of $10 \mathrm{a}_{0} \times 10 \mathrm{a}_{0} \times 50 \mathrm{a}_{0}$.

- The Si PKA energy is $10 \mathrm{keV}$, and temperature is $200 \mathrm{~K}$.

- Each cascade is ended at 10 ps, following by another 10 ps to control temperature.

- Total 140 cascades are simulated, and dose rate is about $10^{8} \mathrm{dpa} / \mathrm{s}$.
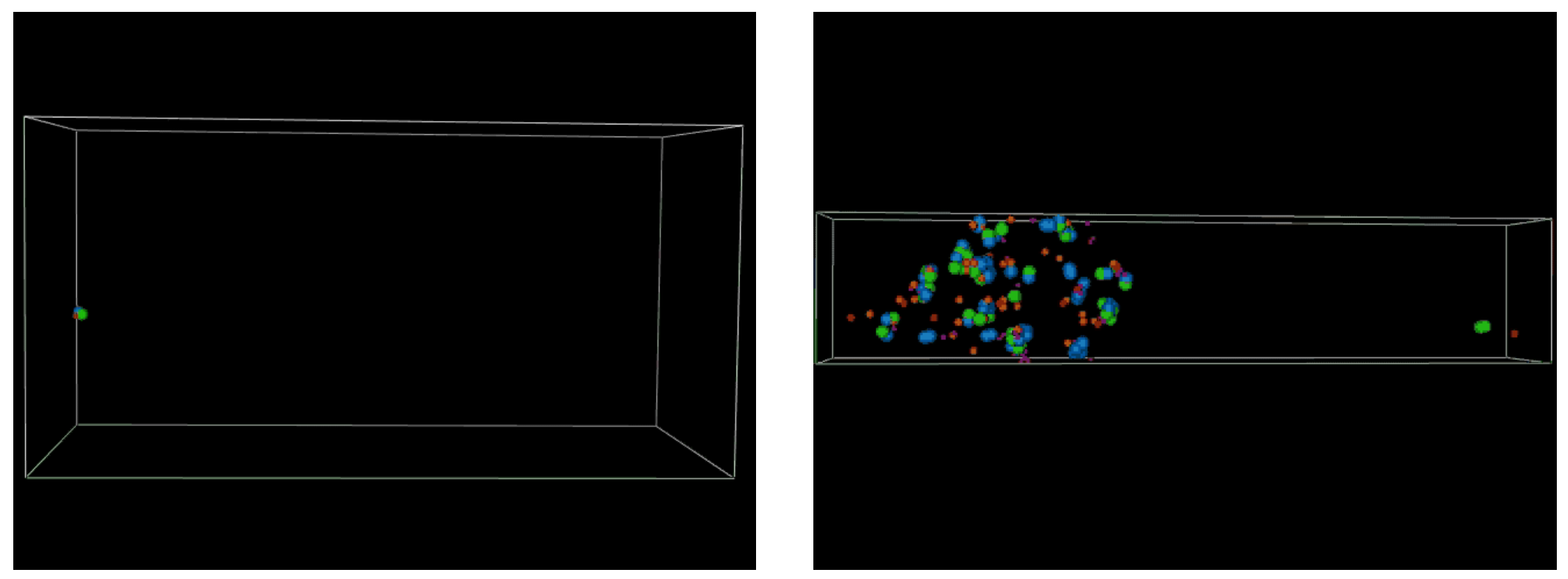


\section{Primary Damage in Ceramics}

\section{Cascade overlap and defect accumulation}

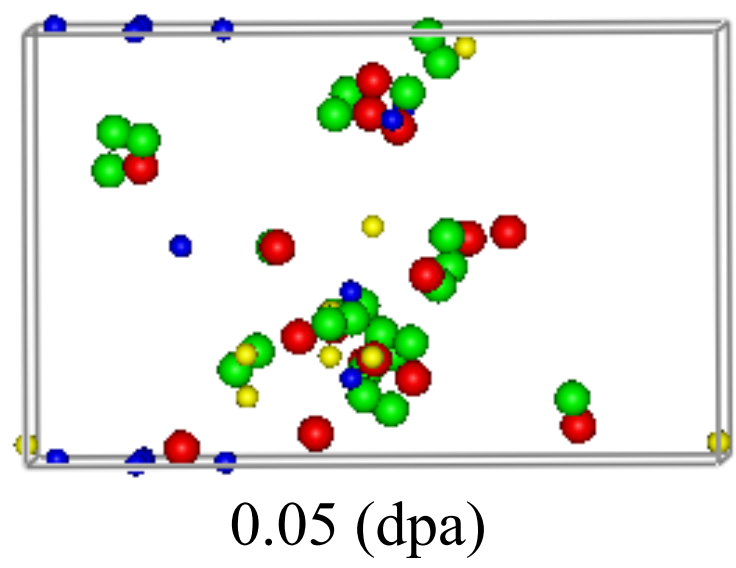

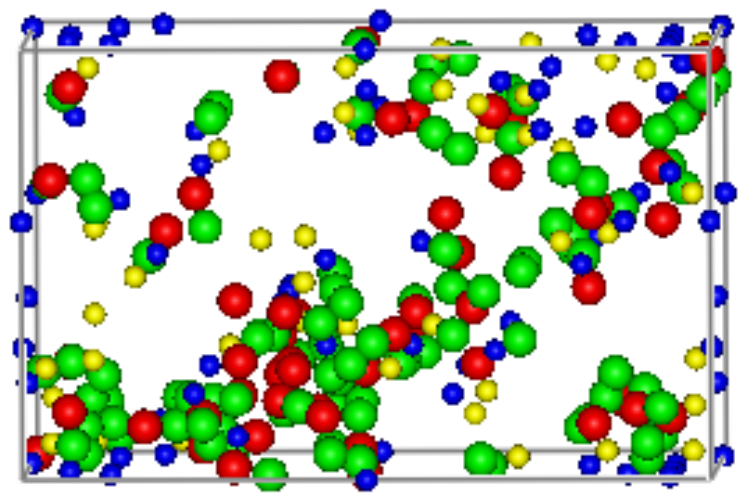

0.10 (dpa)

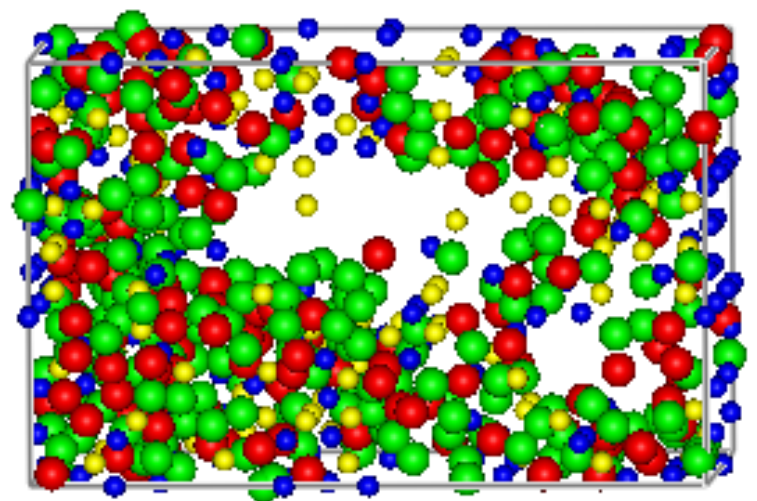

0.20 (dpa)

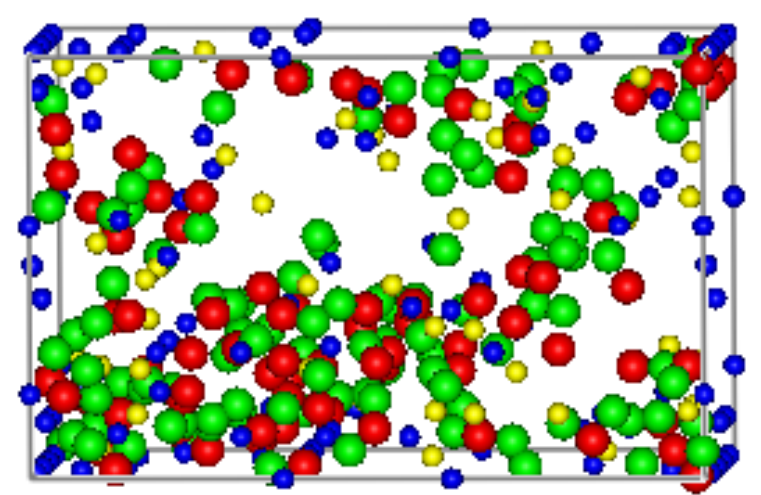

0.13 (dpa)

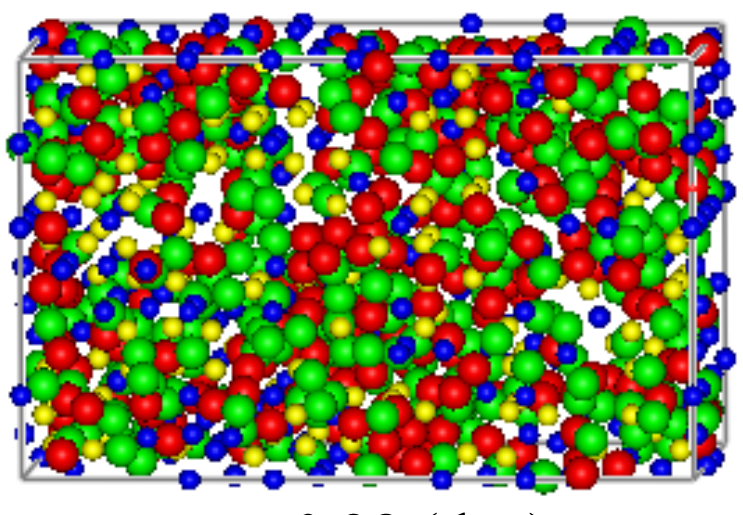

0.28 (dpa)

$\rightarrow$ Both interstitials and antisites are accumulated during overlap process.

$\rightarrow$ Small clusters nucleate at low dose and grow into larger clusters.

$\rightarrow$ The completely disordered state $(0.28 \mathrm{dpa})$ - homogenous distribution of defects. 


\section{Comparison with Experiments}

\section{$\square$ Relative disorder}

$\rightarrow$ Both $\mathrm{C}$ and Si relative disorder increase with increasing dose, showing a sigmoidal dependence on dose.

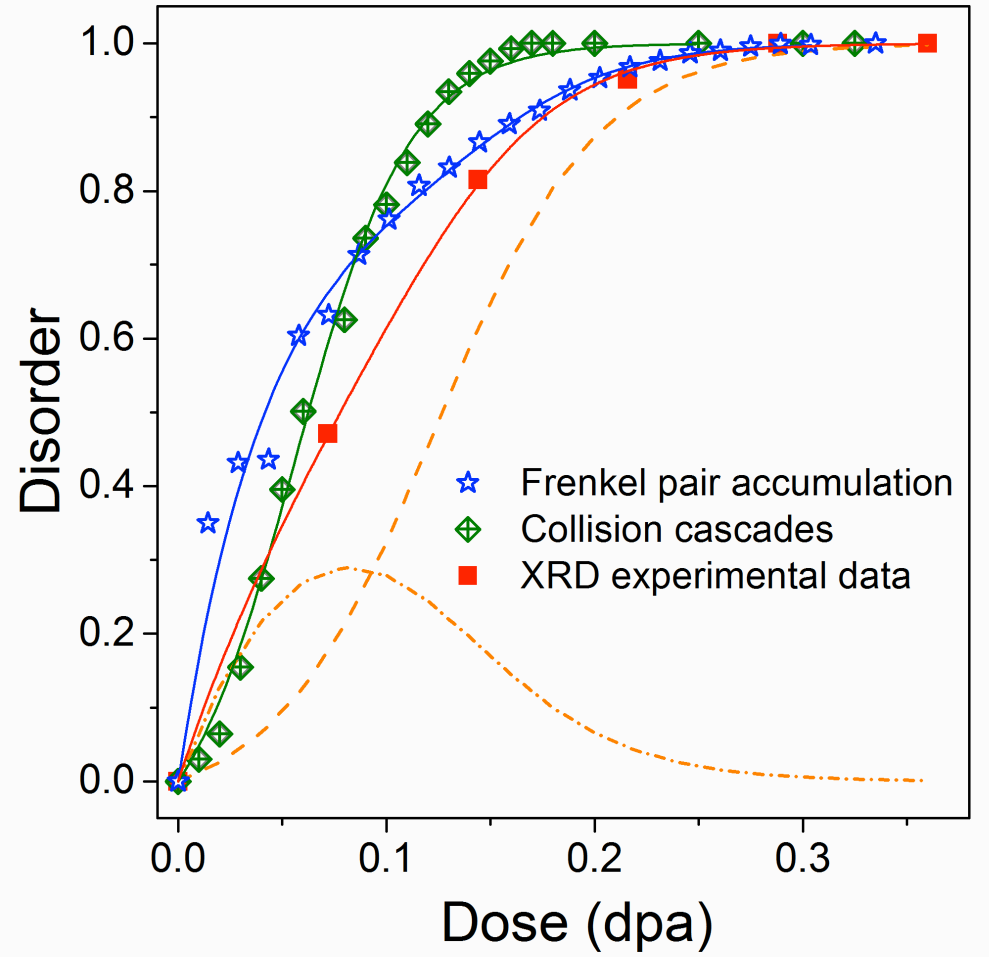

A. Debelle, A. Boulle, A. Chartier, F. Gao, and W. J. Weber, PHYSICAL REVIEW B 90, 174112 (2014)

$\rightarrow$ In general, the simulation results are in good agreement with experimental observations.

$\rightarrow$ The increase in relative disorder at low dose is due to single defect accumulation and small cluster nucleation.

$\rightarrow$ The formation of amorphous domains (clusters) contribute to the rapid increase in the relative disorder at higher dose. 


\section{Comparison with Experiments}

\section{Experimental HRTEM Images}

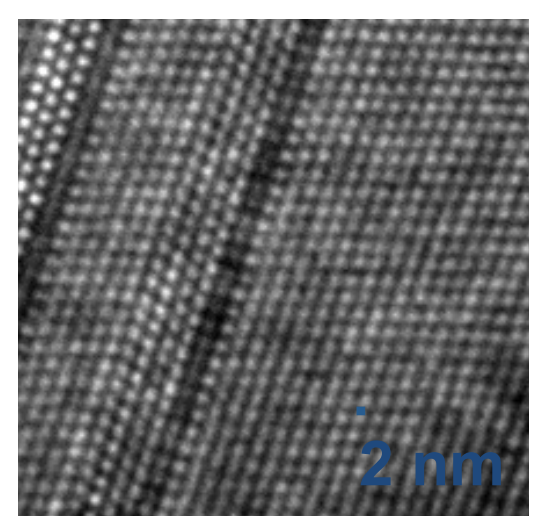

Unirradiated

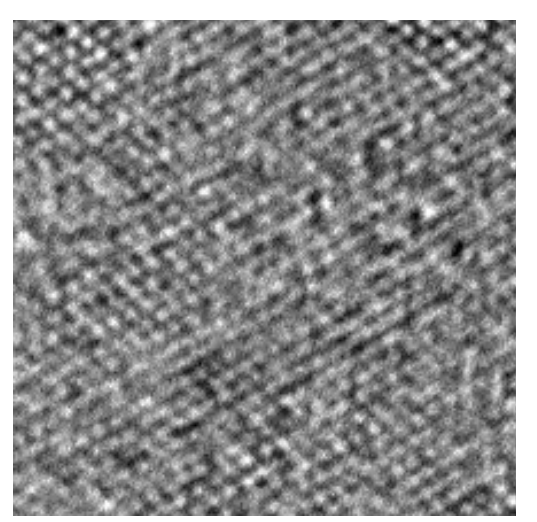

$0.08 \mathrm{dpa}$

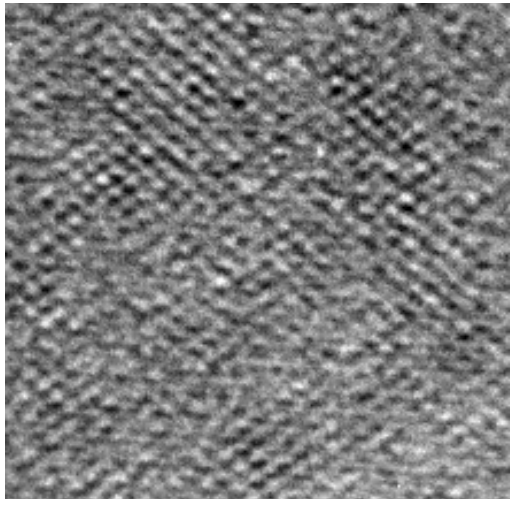

0.16 dpa

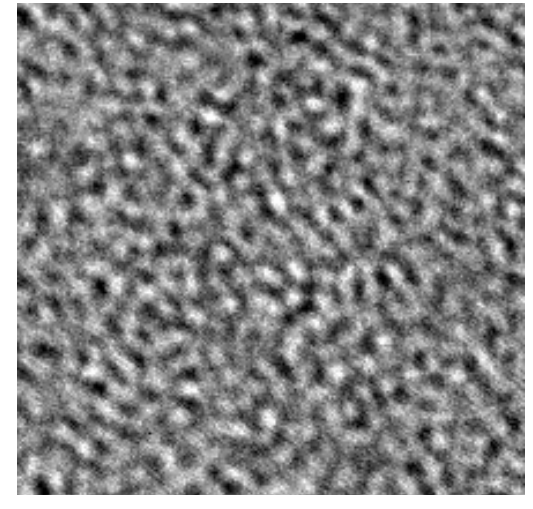

$0.45 \mathrm{dpa}$

HRTEM Image Simulation of Overlapped MD Cascades

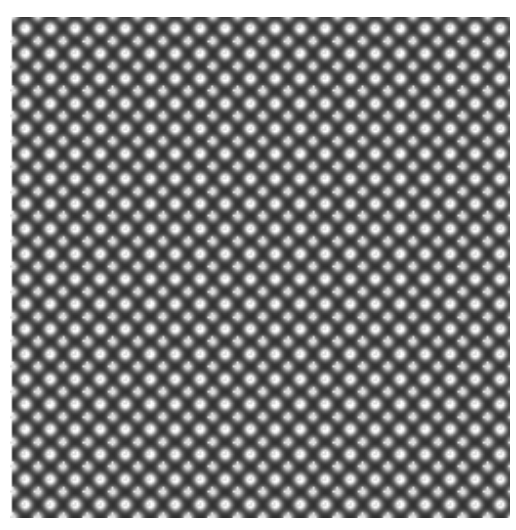

$\mathrm{SiC}$

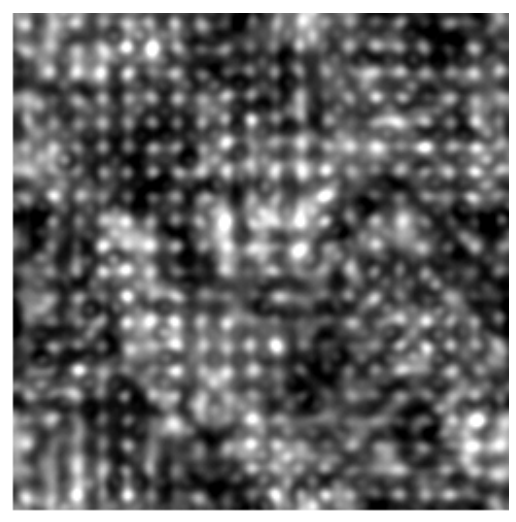

$0.06 \mathrm{dpa}$

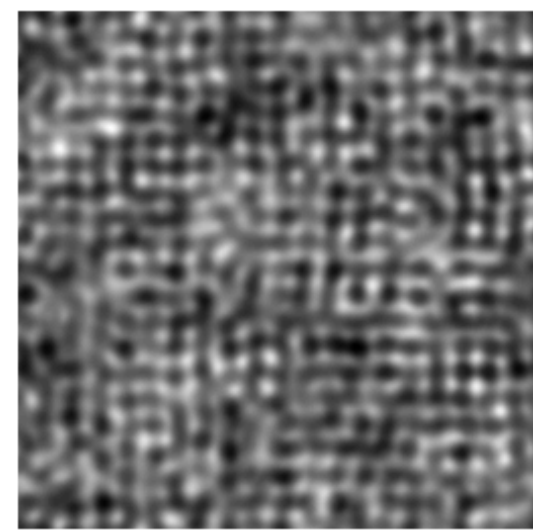

$0.13 \mathrm{dpa}$

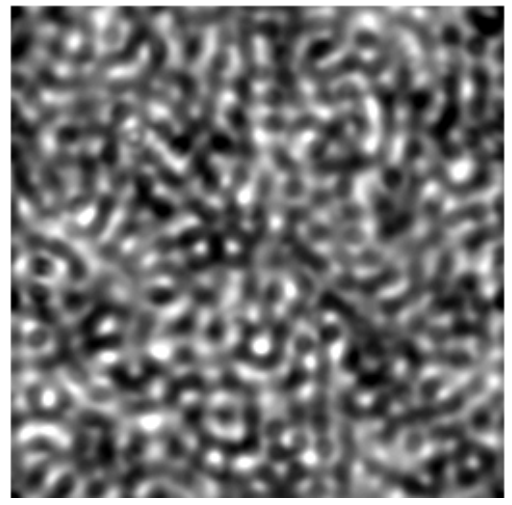

$0.28 \mathrm{dpa}$ 


\title{
Volume Change From MD Cascade Overlap
}

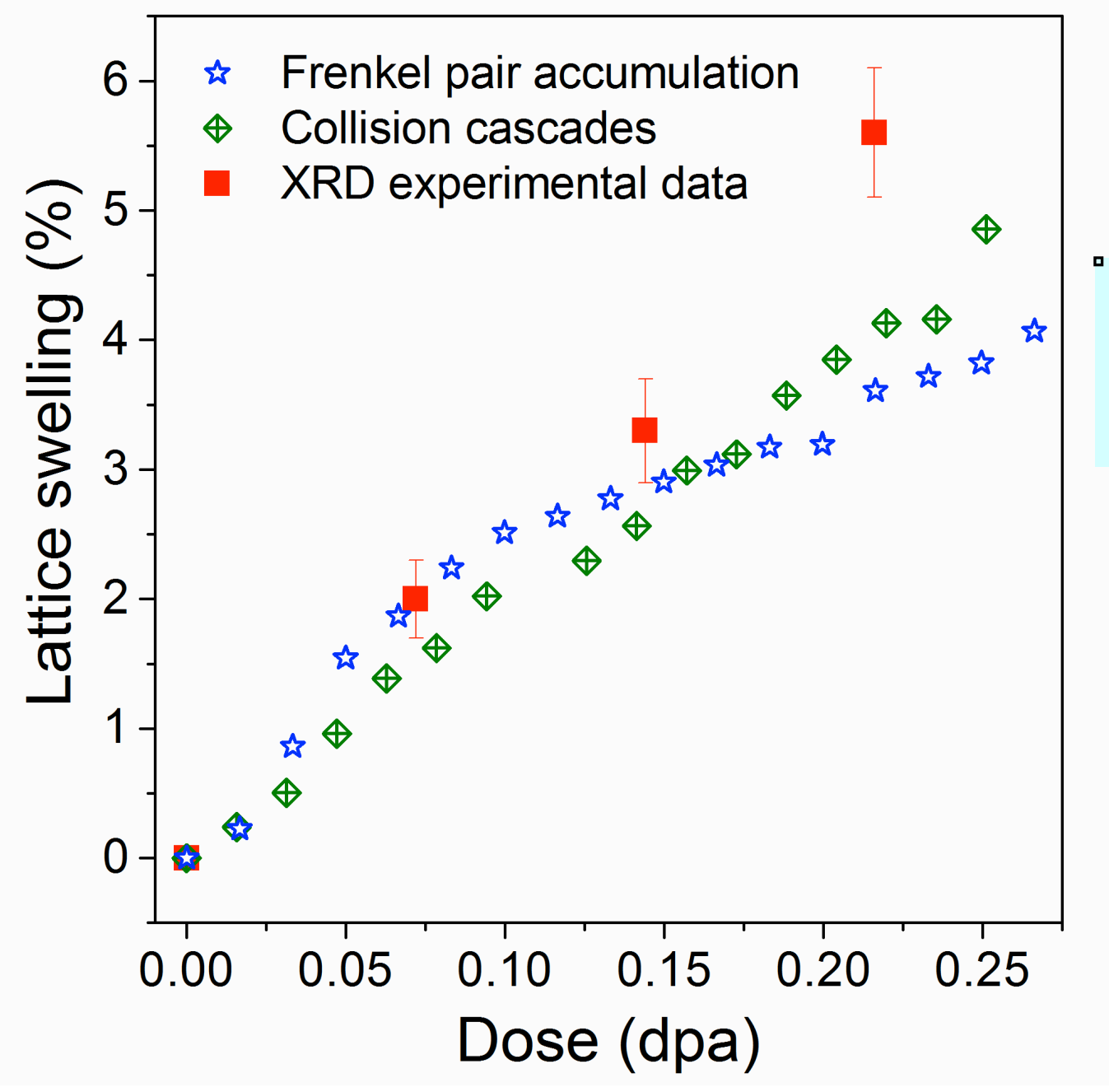

\author{
A. Debelle, A. Boulle, A. \\ Chartier, F. Gao, and W. \\ J. Weber, PHYSICAL \\ REVIEW B 90, 174112 \\ (2014)
}

Experimentally,

(Snead and Hay, 1999)

Predicted Saturation Swelling is in reasonable agreement with experimental value 


\section{Mechanical Properties due to Cascade Overlap}
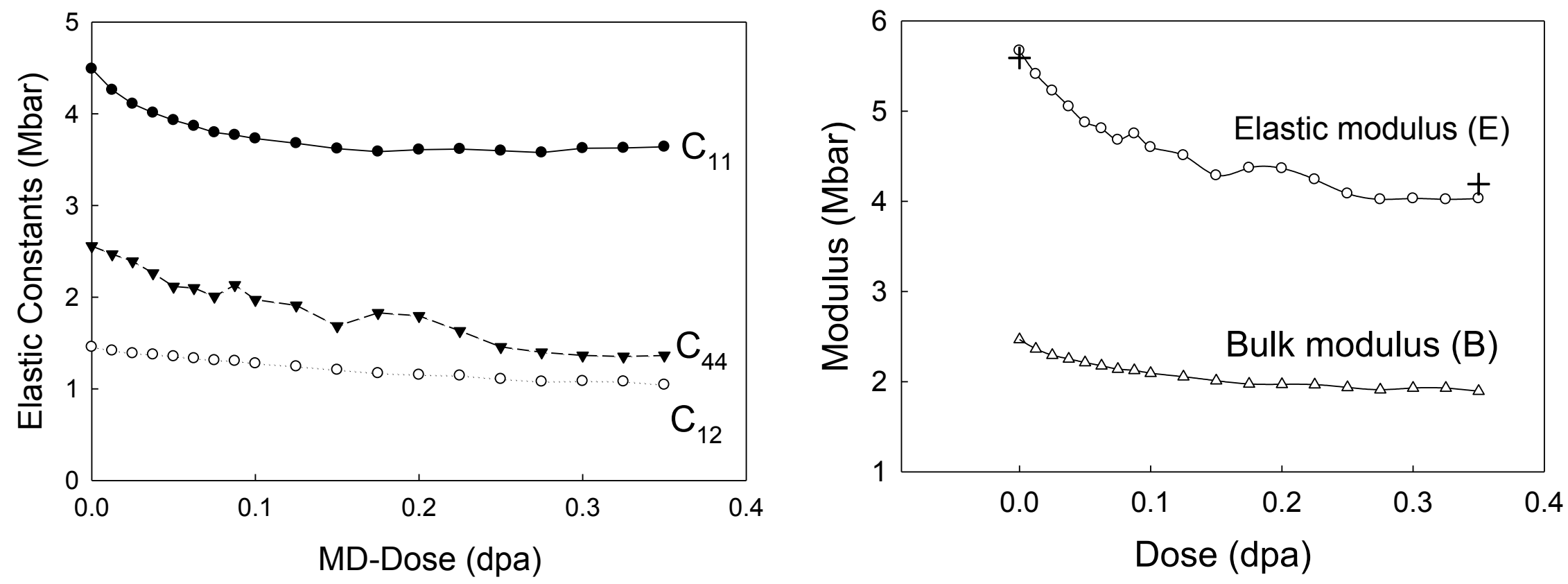

$\rightarrow$ Elastic constants, elastic modulus and bulk modulus decreases with increasing dose.

$\rightarrow$ The reduction in these values is fast at low doses, but slow at high doses.

$\rightarrow$ The most reduction is due to accumulation of point defects and small clusters.

$\rightarrow$ The contribution due to topological disorder associated with amorphous domains is small. 


\section{Mesoscale Simulation of Defect Evolution}

$\square \quad$ Kinetic Monte Carlo Approach
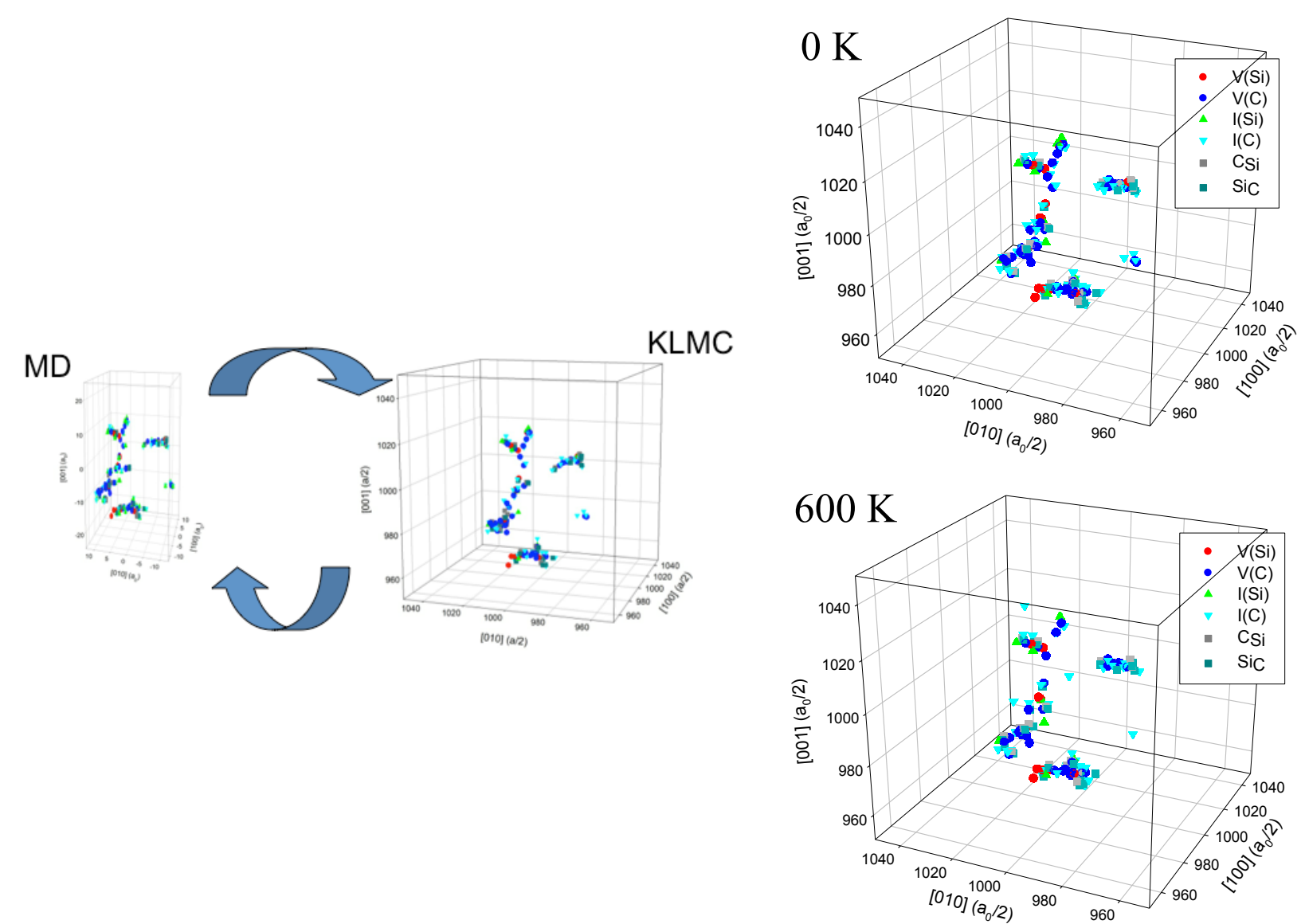
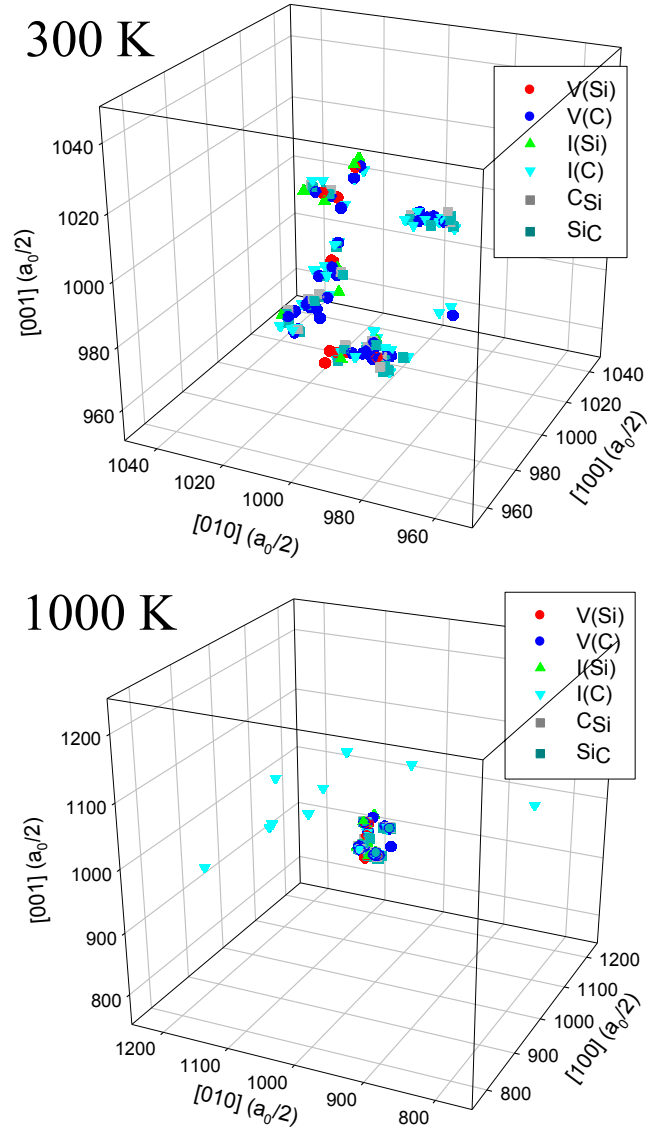

$>$ The total numbers of point defects decreases with increasing temperature. 


\section{Mesoscale Simulation of Defect Evolution}

$\square$ Phase Field Model of Gas Bubble Growth in $\mathrm{UO}_{2}$



Time $=0.002 \mathrm{t}^{*}$

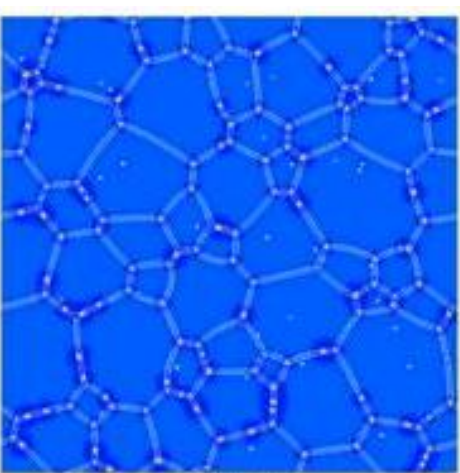

$0.01 t^{*}$

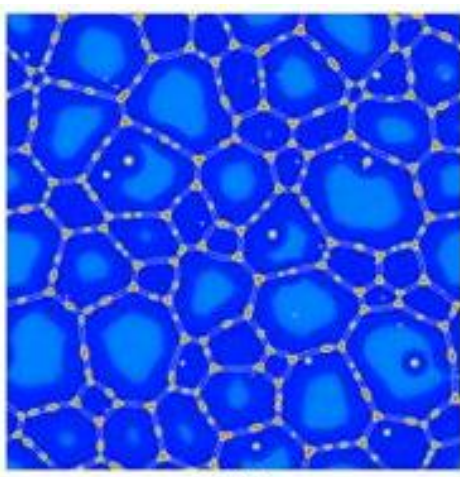

$3.0 t^{*}$

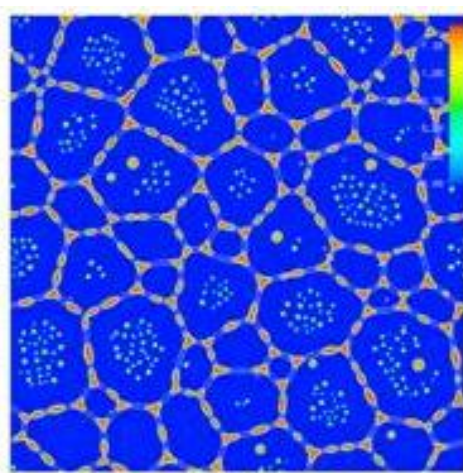

$7.0 \mathrm{t}^{*}$

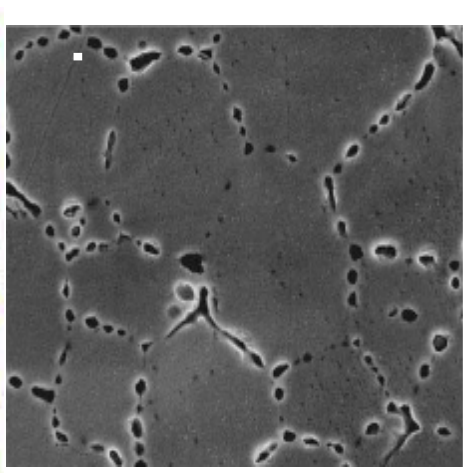

Experiment: Gas bubbles

Simulation: Temporal evolution of gas bubbles (the color denotes gas concentration)

The model takes into account:

$\square$ Diffusion of He atoms and He-V clusters

$\square$ Structural defects

$\square$ Elastic interaction

$\square$ Inhomogeneity of diffusivity

$\square$ Inhomogeneity of elasticity
The model can predict:

$\square$ Gas bubble evolution

$\square$ Gas release

Thermal conductivity evolution

$\square$ Mechanical property evolution 


\section{Mesoscale Simulation of Defect Evolution}

\section{$\square$ Cluster Dynamics Simulation of Void Swelling in Fe-Cr Alloy}

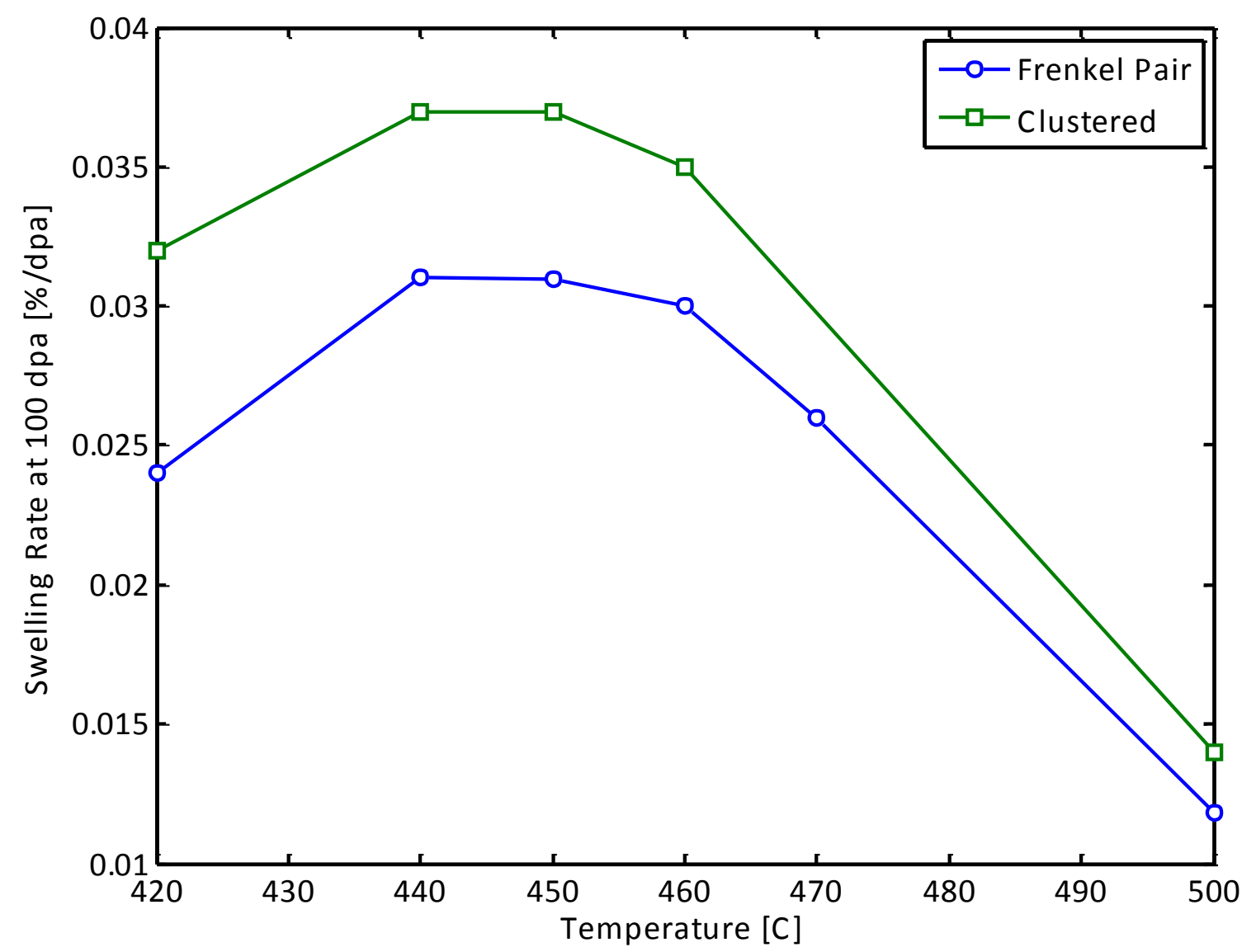

$>$ Effect of Cascade Vacancy Clustering 


\section{SUMMARY}

- Multi-scale approach represents a great tool for studying materials behavior under various conditions.

- Ab initio MD can be used to address charge transfer and electron excitation

- Computer simulations are useful to design new radiation tolerance materials

- The methods to simulating long-time defect evolution need to be further explored

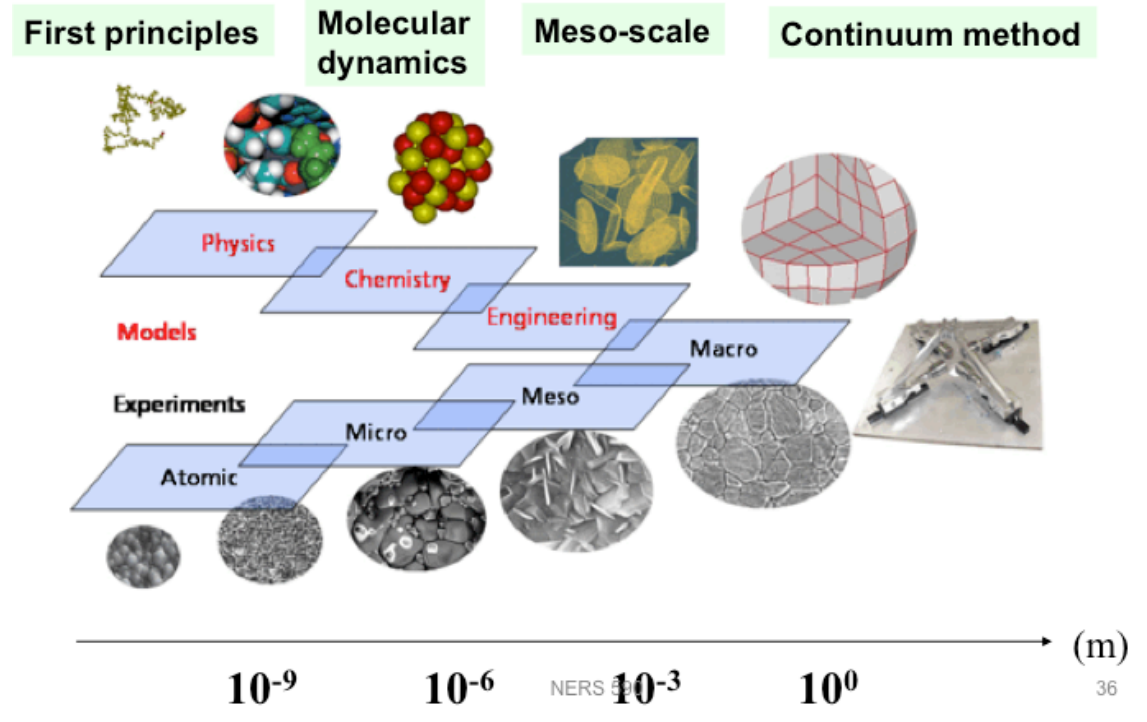

